\title{
Alcohol, tobacco and paan use and understanding of oral cancer risk among Asian males in Leicester
}

\author{
A. R. Vora, ${ }^{1}$ C. M. Yeoman, ${ }^{2}$ and J. P. Hayter, ${ }^{3}$
}

\author{
Objectives To determine use of alcohol, tobacco and paan \\ among males from the various Asian communities in Leicester; \\ and assess their knowledge and attitudes towards oral cancer risk \\ factors and prevention. Also, to determine any differences \\ regarding habits and attitudes between first and second \\ generation Asians.
}

Design Volunteers completed a confidential, bilingual questionnaire regarding alcohol, tobacco and paan use and also knowledge about oral cancer risk factors and preventive measures. Setting Participants were recruited from sources that included GPs' surgeries, sixth form colleges and places of worship.

Subjects Asian males, i.e. those of Indian, Pakistani,

Bangladeshi or Sri Lankan origin; over the age of 16 years and resident in Leicester.

Main outcome measures Quantitative figures were obtained from the questionnaires as to the frequency of alcohol, tobacco and paan use and responses regarding oral cancer knowledge, risk factors and preventive measures.

Results The principal Asian community groups in Leicester were Hindu, Sikh, Muslim and Jain. Significant differences were found in males from these groups with regards to habits and oral cancer awareness. Muslim males use tobacco and paan more than the other groups but avoid alcohol. Sikh males drink more alcohol (especially spirits) than the other groups but their use of tobacco and paan is low. Habits of Hindu and Jain males are variable. However, approximately $10 \%$ of both 1 st and 2 nd generation Hindu males combine all three habits of alcohol, tobacco and paan; and are thus considered to be at high risk of developing oral cancer.Seven percent of 1 st generation Hindu males were found to chew paans containing tobacco which are strongly associated with oral cancer. More 2nd generation Jains drank alcohol than the 1st generation, and a greater proportion of Hindu, Sikh and Jain 2nd generation males drink spirits than their older counterparts. Knowledge of oral cancer risk factors and preventive measures were variable, the lowest level of knowledge being among the 1st generation Sikh group. Few volunteers realised the risk of alcohol drinking in the aetiology of oral cancer.

Conclusion The 'Asian' community in Leicester is not homogeneous, but consists of distinct community groups; each with their own cultural beliefs, habits and attitudes. Knowledge of these differences can be used to provide appropriate health education programmes suitably targeted to reduce the use of the known risk factors for oral cancer.

${ }^{1}$ Research Associate, School of Clinical Dentistry, University of Sheffield, Claremont Crescent, Sheffield S10 2TA ${ }^{2}$ Consultant Oral Physician and Lecturer, Department of Oral and Maxillofacial Surgery,School of Clinical Dentistry, University of Sheffield, Claremont Crescent, Sheffield S10 $2 T^{3}{ }^{3}$ Consultant Maxillofacial Unit, Leicester Royal Infirmary NHS Trust, Leicester LE1 5WW. ${ }^{*}$ Correspondence to J. P. Hayter

REFEREED PAPER

Received 14.06.99; accepted 29.11.99

(c) British Dental Journal 2000; 188: 444-451
$\mathrm{U}^{\mathrm{p}}$ to $75 \%$ of oral cavity cancers, ${ }^{1}$ the majority of which are squamous cell carcinomas, are thought to be attributable to the separate and combined habits of alcohol drinking, tobacco use and paan (betel-quid) chewing. ${ }^{2-4}$

In the West, the principal known aetiological factors for oral cancer are tobacco use and alcohol drinking. Epidemiological studies have shown a strong association between the use of tobacco (smoked and chewed) and oral carcinogenesis. ${ }^{5-7}$ However, alcohol is increasingly being recognised as an important risk factor, having a multiplicative synergistic carcinogenic effect with tobacco. ${ }^{2,8-10}$ It has been calculated that a non-drinking heavy smoker (smoking more than twenty per day ${ }^{11}$ ) may have a two- to four-fold increased risk of developing squamous cell carcinoma than a non-smoker, whereas a heavy smoker and drinker (drinking over 21 units/week) ${ }^{12}$ has a six- to fifteen-times increased risk. ${ }^{1,13,14}$

However, the other known risk habit of paan chewing is widespread in the Indian sub-continent, ${ }^{4}$ where oral cancer accounts for more than one third of all cancers compared with the prevalence of $3 \%$ of all malignancies in Western Europe. Paan use is also prevalent among Asian communities living in the United Kingdom; ${ }^{15,16}$ and a higher number of oral and pharyngeal cancers within immigrants of Indian ethnic origin compared with Caucasians has been reported. ${ }^{17-21}$

The components of paan can vary between communities and individuals, ${ }^{22}$ although the major constituents are the leaf of Piper betel (betel leaf); sliced nut of Areca catechu (areca nut/sopari); lime; catechu (a resinous extract from the wood of the acacia tree); grated coconut and a variety of spices such as aniseed, peppermint, cardamom and cloves. ${ }^{15,23}$ The addition of tobacco to paan is associated with a significant risk of oral cancer development. The presence of lime within paan has also been shown to release reactive oxygen species from extracts of areca nut that could contribute to the cytogenetic damage involved in the aetiology of oral cancer. ${ }^{24} \mathrm{~A}$ synergistic increase in oral cancer risk has been shown among people who consume alcohol, smoke and chew paan. ${ }^{4,25,26}$

In addition, the chewing of areca nut is associated with the premalignant condition of oral submucous fibrosis especially when used in the form of paan masala. ${ }^{4,27,28}$ Paan masala (or guthkha) is a dry complex mixture, with or without tobacco, of areca nut, catechu, lime, cardamom, flavourings and sweetening agents; and is sold and readily available in the UK. Paan masala has been shown to be genotoxic, causing DNA damage via water soluble direct acting mutagens such as the areca nut alkaloids (arecoline, guvacoline, arecadine, guvacine) and tobacco derived nitrosamines found within the mixtures; with mutagenicity being potentiated by the use of ethanol. ${ }^{23,29}$

Previous epidemiological studies of cancer incidence and mortality classify 'Asians' together as one group when making comparisons with the Caucasian population. ${ }^{18,30}$ However, the Asian population in the UK is not a culturally homogeneous group, but consists of a number of distinctly separate communities with dif- 
Table 1 Age and community group structure of the Asian male participants of the study

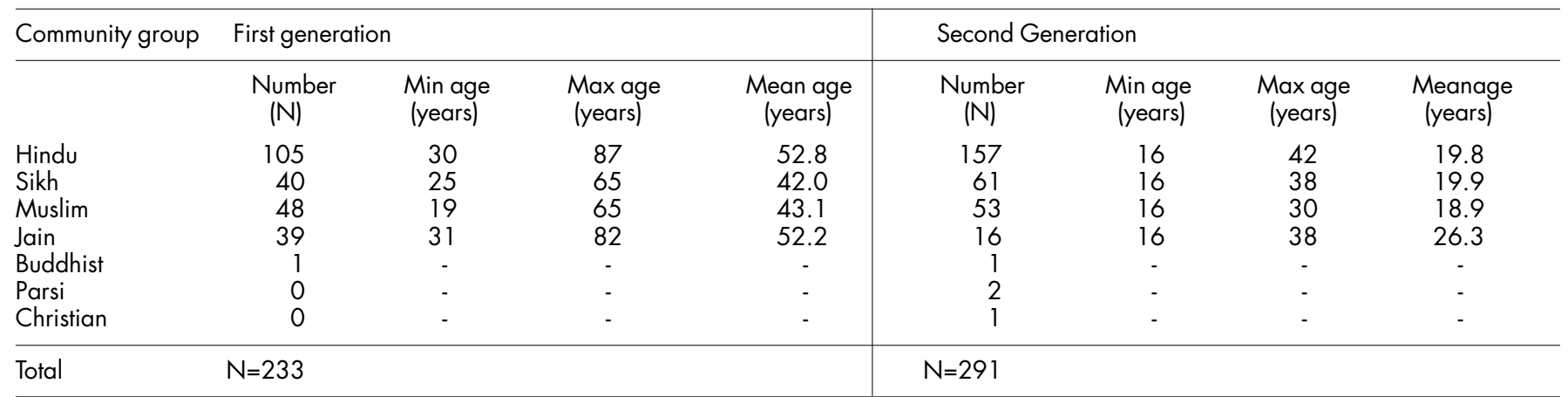

ferences in religious beliefs, diet and the partaking of habits such as alcohol drinking, tobacco smoking and paan chewing. ${ }^{11,31-33}$

Differences in attitude to these values can also vary between first generation Asian immigrants compared with those of second generation Asians (born and/or educated and living in the United Kingdom). ${ }^{34,35}$

Leicester is a multicultural city with a significant Asian population comprising of people from India, Sri Lanka, Bangladesh, Pakistan and East Africa. ${ }^{36,37}$ Since the lifestyle of many of these people is influenced by religious beliefs and values, ${ }^{11,33}$ the aim of this study was to determine the frequency of alcohol, tobacco and paan use; and assess knowledge and attitudes towards oral cancer risk among Hindu, Sikh, Muslim and Jain males living in Leicester. In addition, the existence of any differences regarding habits and attitude towards oral cancer between first and second generation males in each community group was to be assessed.

\section{Subjects and Methods}

Ethical approval for the study was obtained from the Leicestershire Ethics Committee.

Recruitment of subjects was voluntary. Asian males over the age of 16 years were recruited from GP surgeries, sixth form colleges, places of worship and shopping centres.

Participants read a sheet outlining details of the study, gave consent, and completed a confidential bilingual questionnaire written in English and Gujarati (a language spoken by approximately two- thirds of the Asian population in Leicester). Questions covering age; ethnic origin; religious belief; use of alcohol, tobacco, paan, areca nut and paan masala; and knowledge of oral cancer risk and prevention were answered by a combination of ticking relevant boxes and filling in blank spaces on the form.

A total of 524 correctly completed questionnaires were obtained. These were initially separated according to religion; and then into first or second generation which was assessed using place of birth and length of time resident in the United Kingdom. A person was considered to be a second generation Asian if they were born and lived in the United Kingdom for all of their life; or, if born outside the United Kingdom, had been living in the country before the age of 16 years and had received primary and secondary school education in the United Kingdom.

No computer databases were used to store the data obtained, with information being manually transferred and summarised from the questionnaires onto tables. Statistical analysis for significance for the differences observed in the contingency table data produced in this study was performed by the use of the $\chi^{2}$ test, using the null hypothesis that no difference was to be expected between the various groups.

Table 1 outlines the age and community group structure of the Asian male participants of the study. Large enough sample sizes for comparison were available for the Hindu, Sikh, Muslim and Jain groups, which have been shown to be the main religions of Leicester's Asian population. ${ }^{37}$

Table 2 Alcohol use among Leicester Asian males in the study group

\begin{tabular}{|c|c|c|c|c|c|c|c|c|c|c|c|c|c|}
\hline \multirow[t]{2}{*}{ Sample group } & \multicolumn{3}{|c|}{ Alcohol use } & \multicolumn{4}{|c|}{ Units/week in drinkers } & \multicolumn{6}{|c|}{ Type of alcohol drunk by drinkers } \\
\hline & No & Occasional & Yes & Max & Min & Mean & St.dev. & Beer/Lager & Spirits & Cider & Wine & Alcopop & Other \\
\hline \multicolumn{14}{|c|}{ First generation } \\
\hline Hindu $(n=105)$ & $\begin{array}{r}43 \\
41 \%\end{array}$ & $\begin{array}{c}21 \\
20 \%\end{array}$ & $\begin{array}{c}41 \\
39 \%\end{array}$ & 40 & 1 & 10.3 & 8.9 & $\begin{array}{r}51 / 62 \\
82 \%\end{array}$ & $\begin{array}{r}18 / 62 \\
29 \%\end{array}$ & $\begin{array}{r}3 / 62 \\
5 \%\end{array}$ & $\begin{array}{r}8 / 62 \\
8 \%\end{array}$ & $\begin{array}{r}0 / 62 \\
0 \%\end{array}$ & $\begin{array}{r}0 / 62 \\
0 \%\end{array}$ \\
\hline Sikh $(n=40)$ & $\begin{array}{r}15 \\
37 \%\end{array}$ & $\begin{array}{r}8 \\
20 \%\end{array}$ & $\begin{array}{r}17 \\
43 \%\end{array}$ & 40 & 2 & 12.8 & 9.7 & $\begin{array}{r}22 / 25 \\
88 \%\end{array}$ & $\begin{array}{r}13 / 25 \\
52 \%\end{array}$ & $\begin{array}{r}0 / 25 \\
0 \%\end{array}$ & $\begin{array}{r}1 / 25 \\
4 \%\end{array}$ & $\begin{array}{r}0 / 25 \\
0 \%\end{array}$ & $\begin{array}{r}0 / 25 \\
0 \%\end{array}$ \\
\hline Muslim (n=48) & $\begin{array}{r}44 \\
92 \%\end{array}$ & $\begin{array}{r}2 \\
4 \%\end{array}$ & $\begin{array}{r}2 \\
4 \%\end{array}$ & 5 & 4 & 4.3 & 0.6 & $\begin{array}{l}2 / 4 \\
50 \%\end{array}$ & $\begin{array}{l}2 / 4 \\
50 \%\end{array}$ & $\begin{array}{r}0 / 4 \\
0 \%\end{array}$ & $\begin{array}{l}1 / 4 \\
25 \%\end{array}$ & $\begin{array}{r}0 \\
0 \%\end{array}$ & $\begin{array}{r}0 \\
0 \%\end{array}$ \\
\hline Jain $(n=39)$ & $\begin{array}{r}32 \\
82 \%\end{array}$ & $\begin{array}{r}2 \\
5 \%\end{array}$ & $\begin{array}{r}5 \\
13 \%\end{array}$ & 20 & 1 & 4.8 & 8.5 & $\begin{array}{l}3 / 7 \\
43 \%\end{array}$ & $\begin{array}{l}1 / 7 \\
14 \%\end{array}$ & $\begin{array}{r}0 / 7 \\
0 \%\end{array}$ & $\begin{array}{l}4 / 7 \\
57 \%\end{array}$ & $\begin{array}{r}0 / 7 \\
0 \%\end{array}$ & $\begin{array}{r}0 / 7 \\
0 \%\end{array}$ \\
\hline \multicolumn{14}{|c|}{ Second generation } \\
\hline Hindu $(n=157)$ & $\begin{array}{r}47 \\
30 \%\end{array}$ & $\begin{array}{r}40 \\
25 \%\end{array}$ & $\begin{array}{r}70 \\
45 \%\end{array}$ & 58 & 1 & 7.8 & 10.6 & $\begin{array}{r}74 / 110 \\
67 \%\end{array}$ & $\begin{array}{r}57 / 110 \\
52 \%\end{array}$ & $\begin{array}{r}22 / 110 \\
20 \%\end{array}$ & $\begin{array}{r}11 / 110 \\
10 \%\end{array}$ & $\begin{array}{r}21 / 110 \\
19 \%\end{array}$ & $\begin{array}{r}1 / 110 \\
1 \%\end{array}$ \\
\hline Muslim (n=53) & $\begin{array}{r}50 \\
94 \%\end{array}$ & $\begin{array}{c}1 \\
2 \%\end{array}$ & $\begin{array}{r}2 \\
4 \%\end{array}$ & 42 & 2 & 18 & 21.2 & $\begin{array}{l}2 / 3 \\
67 \%\end{array}$ & $\begin{array}{l}2 / 3 \\
67 \%\end{array}$ & $\begin{array}{r}0 / 3 \\
0 \%\end{array}$ & $\begin{array}{r}0 / 3 \\
0 \%\end{array}$ & $\begin{array}{r}1 / 3 \\
0 \%\end{array}$ & $\begin{array}{r}0 / 3 \\
0 \%\end{array}$ \\
\hline Jain $(n=16)$ & $\begin{array}{r}6 \\
37 \%\end{array}$ & $\begin{array}{r}3 \\
19 \%\end{array}$ & $\begin{array}{r}7 \\
44 \%\end{array}$ & 25 & 1 & 8.5 & 9.5 & $\begin{array}{r}8 / 11 \\
73 \%\end{array}$ & $\begin{array}{r}3 / 11 \\
27 \%\end{array}$ & $\begin{array}{r}1 / 11 \\
9 \%\end{array}$ & $\begin{array}{r}2 / 11 \\
27 \%\end{array}$ & $\begin{array}{r}2 / 11 \\
27 \%\end{array}$ & $\begin{array}{r}0 / 11 \\
0 \%\end{array}$ \\
\hline
\end{tabular}


RESEARCH

oral cancer

Table 3 Tobacco use among Leicester Asian males in the study group

\begin{tabular}{|c|c|c|c|c|c|c|c|c|}
\hline \multirow[t]{2}{*}{ Sample group } & \multicolumn{3}{|c|}{ Tobacco use } & \multicolumn{2}{|c|}{$\begin{array}{l}\text { No. of } \\
\text { cigarette smokers }\end{array}$} & \multicolumn{2}{|c|}{ Cigarettes/day } & \multirow[b]{2}{*}{ St.dev } \\
\hline & No & Occasional & Yes & & Min & Max & Mean & \\
\hline \multicolumn{9}{|c|}{ First generation } \\
\hline Hindu $(n=105)$ & $\begin{array}{r}85 \\
81 \%\end{array}$ & $\begin{array}{r}2 \\
2 \%\end{array}$ & $\begin{array}{r}18 \\
17 \%\end{array}$ & $\begin{array}{r}16 / 20 \\
80 \%\end{array}$ & 4 & 30 & 10.1 & 6.3 \\
\hline Sikh $(n=40)$ & $\begin{array}{r}38 \\
95 \%\end{array}$ & $\begin{array}{r}1 \\
2.5 \%\end{array}$ & $\begin{array}{r}1 \\
2.5 \%\end{array}$ & $\begin{array}{l}1 / 2 \\
50 \%\end{array}$ & - & - & 100 & \\
\hline Muslim (n=48) & $\begin{array}{r}22 \\
46 \%\end{array}$ & $\begin{array}{r}5 \\
10 \%\end{array}$ & $\begin{array}{r}21 \\
44 \%\end{array}$ & $\begin{array}{r}25 / 26 \\
96 \%\end{array}$ & 1 & 40 & 11.7 & 9.4 \\
\hline Jain $(n=39)$ & $\begin{array}{r}34 \\
87 \%\end{array}$ & $\begin{array}{r}1 \\
3 \%\end{array}$ & $\begin{array}{r}4 \\
10 \%\end{array}$ & $\begin{array}{r}5 / 5 \\
100 \%\end{array}$ & 1 & 7 & 4 & 2.5 \\
\hline \multicolumn{9}{|c|}{ Second generation } \\
\hline Hindu $(n=157)$ & $\begin{array}{l}115 \\
73 \%\end{array}$ & $\begin{array}{l}11 \\
7 \%\end{array}$ & $\begin{array}{r}31 \\
20 \%\end{array}$ & $\begin{array}{r}40 / 42 \\
95 \%\end{array}$ & 1 & 18 & 5.9 & 4.3 \\
\hline Sikh $(n=61)$ & $\begin{array}{r}52 \\
85 \%\end{array}$ & $\begin{array}{r}2 \\
3 \%\end{array}$ & $\begin{array}{r}7 \\
12 \%\end{array}$ & $\begin{array}{r}9 / 9 \\
100 \%\end{array}$ & 1 & 15 & 8.3 & 4.7 \\
\hline Muslim (n=53) & $\begin{array}{r}37 \\
70 \%\end{array}$ & $\begin{array}{r}6 \\
11 \%\end{array}$ & $\begin{array}{r}10 \\
19 \%\end{array}$ & $\begin{array}{r}15 / 16 \\
94 \%\end{array}$ & 1 & 10 & 5.9 & 3.6 \\
\hline Jain $(n=16)$ & $\begin{array}{r}14 \\
87.5 \%\end{array}$ & $\begin{array}{r}0 \\
0 \%\end{array}$ & $\begin{array}{r}2 \\
12.5 \%\end{array}$ & $\begin{array}{r}2 / 2 \\
100 \%\end{array}$ & 10 & 20 & 15 & 7.1 \\
\hline
\end{tabular}

\section{Results}

Alcohol Use

Statistically significant differences were found between the different community groups with regards to the drinking of alcohol in both 1 st and 2 nd generation Asians $\left(\chi^{2}, p<0.001\right)$ (Fig. 1, Table 2).

Alcohol use was principally found in the Sikh and Hindu com-

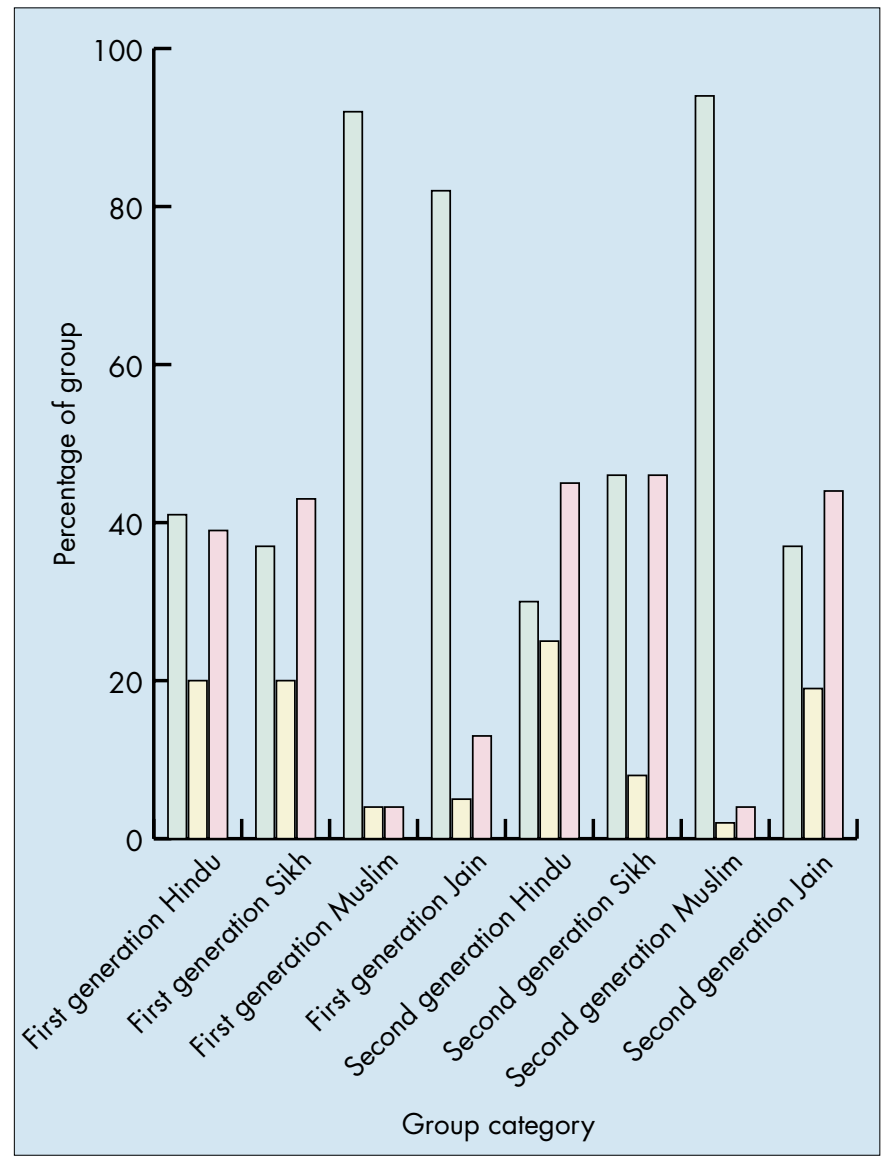

Fig. 1 Alcohol drinking by Leicester Asian males in study sample. Green, non-drinkers; yellow, occasionally drink; red, regular drinkers. munities, with highest alcohol consumption among 1st generation Asians being observed in the Sikh community $\left(\chi^{2}, p<0.001\right)$. Forty three per cent stated that they were regular alcohol drinkers, with a further $20 \%$ drinking alcohol occasionally. Mean units of alcohol drank per week was also highest in the Sikh community than the other 1st generation groups. Levels of drinking by 2 nd

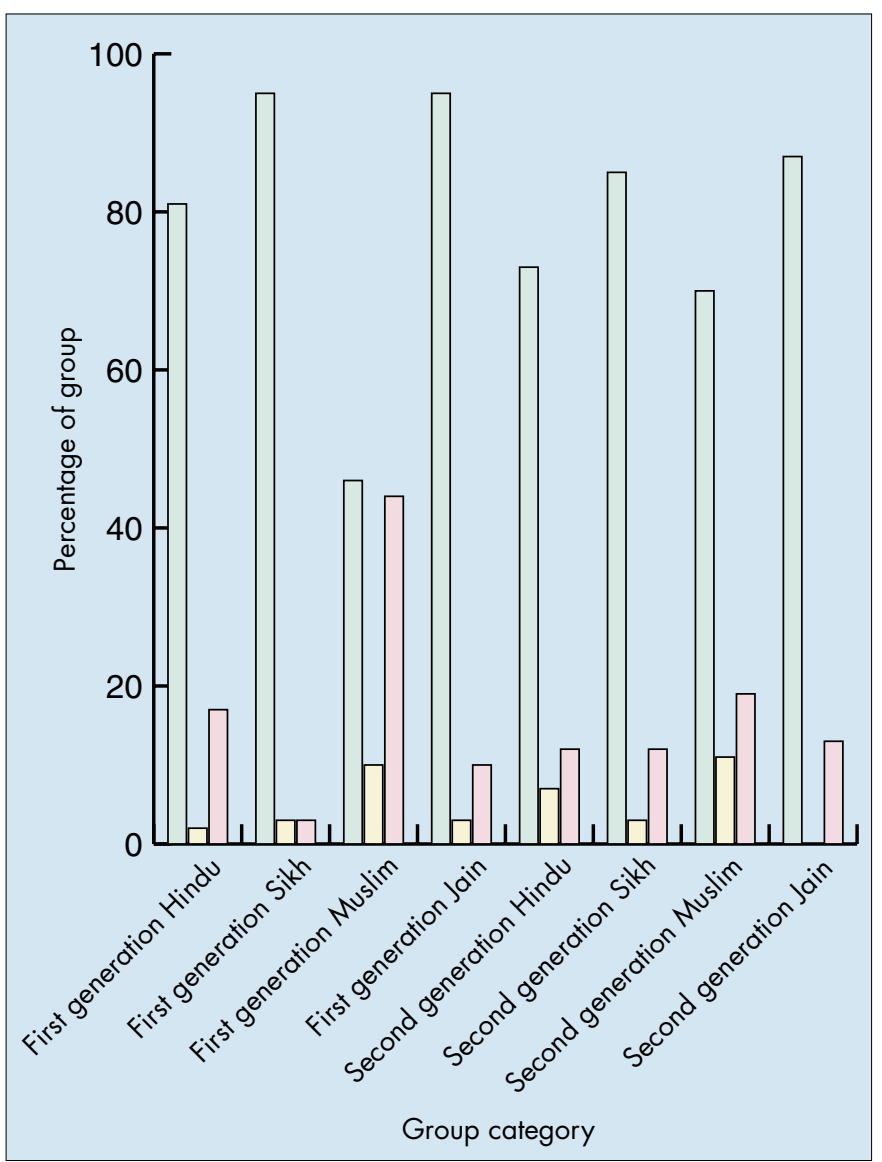

Fig. 2 Tobacco use among Leicester Asian males in study group. Green, non-users; yellow, occasional use; red, regular use. 
Table 4 Paan (Betel Quid) use among Leicester Asian males in the study group

\begin{tabular}{|c|c|c|c|c|c|c|c|c|c|c|c|c|}
\hline \multirow[t]{2}{*}{ Sample group } & \multicolumn{3}{|c|}{ Paan use } & \multicolumn{4}{|c|}{ Paans chewed per week } & \multicolumn{5}{|c|}{ Type of paan chewed } \\
\hline & No & Occasional & Yes & Min & Max & Mean & St.dev. & Sweet & Khari & $\begin{array}{l}\text { With } \\
\text { tobacco }\end{array}$ & Plain leaf & $\begin{array}{l}\text { Not } \\
\text { stated }\end{array}$ \\
\hline \multicolumn{13}{|c|}{ First generation } \\
\hline Hindu $(n=105)$ & $\begin{array}{r}67 \\
64 \%\end{array}$ & $\begin{array}{r}28 \\
27 \%\end{array}$ & $\begin{array}{l}10 \\
9 \%\end{array}$ & 1 & 20 & 4.8 & 5.7 & $\begin{array}{r}13 / 38 \\
34 \%\end{array}$ & $\begin{array}{r}13 / 38 \\
34 \%\end{array}$ & $\begin{array}{r}8 / 38 \\
21 \%\end{array}$ & $\begin{array}{r}2 / 38 \\
6 \%\end{array}$ & $\begin{array}{r}3 / 38 \\
8 \%\end{array}$ \\
\hline Muslim (n=48) & $\begin{array}{r}28 \\
58 \%\end{array}$ & $\begin{array}{r}8 \\
17 \%\end{array}$ & $\begin{array}{r}12 \\
25 \%\end{array}$ & 1 & 40 & 8.8 & 12.2 & $\begin{array}{r}11 / 20 \\
55 \%\end{array}$ & $\begin{array}{r}8 / 20 \\
40 \%\end{array}$ & $\begin{array}{r}1 / 20 \\
5 \%\end{array}$ & $\begin{array}{r}0 / 20 \\
0 \%\end{array}$ & $\begin{array}{r}0 / 20 \\
0 \%\end{array}$ \\
\hline Jain $(n=39)$ & $\begin{array}{r}19 \\
49 \%\end{array}$ & $\begin{array}{r}15 \\
38 \%\end{array}$ & $\begin{array}{r}5 \\
13 \%\end{array}$ & 1 & 5 & 2 & 1.6 & $\begin{array}{r}10 / 20 \\
50 \%\end{array}$ & $\begin{array}{r}9 / 20 \\
45 \%\end{array}$ & $\begin{array}{r}1 / 20 \\
5 \%\end{array}$ & $\begin{array}{r}0 / 20 \\
0 \%\end{array}$ & $\begin{array}{r}1 / 20 \\
5 \%\end{array}$ \\
\hline \multicolumn{13}{|c|}{ Second generation } \\
\hline Hindu $(n=157)$ & $\begin{array}{r}96 \\
61 \%\end{array}$ & $\begin{array}{r}46 \\
29 \%\end{array}$ & $\begin{array}{r}15 \\
10 \%\end{array}$ & 1 & 40 & 2.8 & 6.4 & $\begin{array}{r}51 / 61 \\
84 \%\end{array}$ & $\begin{array}{r}7 / 61 \\
11 \%\end{array}$ & $\begin{array}{r}2 / 61 \\
3 \%\end{array}$ & $\begin{array}{r}0 / 61 \\
0 \%\end{array}$ & $\begin{array}{r}2 / 61 \\
3 \%\end{array}$ \\
\hline Sikh $(n=61)$ & $\begin{array}{r}56 \\
92 \%\end{array}$ & $\begin{array}{r}2 \\
3 \%\end{array}$ & $\begin{array}{r}3 \\
5 \%\end{array}$ & 1 & 70 & 24 & 39.8 & $\begin{array}{r}5 / 5 \\
100 \%\end{array}$ & $\begin{array}{r}0 / 5 \\
0 \%\end{array}$ & $\begin{array}{r}0 / 5 \\
0 \%\end{array}$ & $\begin{array}{r}0 / 5 \\
0 \%\end{array}$ & $\begin{array}{r}0 / 5 \\
0 \%\end{array}$ \\
\hline Muslim (n=53) & $\begin{array}{r}35 \\
66 \%\end{array}$ & $\begin{array}{r}7 \\
13 \%\end{array}$ & $\begin{array}{r}11 \\
21 \%\end{array}$ & 1 & 30 & 4.5 & 8.0 & $\begin{array}{r}16 / 18 \\
89 \%\end{array}$ & $\begin{array}{r}3 / 18 \\
17 \%\end{array}$ & $\begin{array}{r}0 / 18 \\
0 \%\end{array}$ & $\begin{array}{r}0 / 18 \\
0 \%\end{array}$ & $\begin{array}{r}0 / 18 \\
0 \%\end{array}$ \\
\hline Jain $(n=16)$ & $\begin{array}{r}9 \\
56 \%\end{array}$ & $\begin{array}{r}4 \\
25 \%\end{array}$ & $\begin{array}{r}3 \\
19 \%\end{array}$ & 1 & 2 & 1.2 & 0.5 & $\begin{array}{l}5 / 7 \\
71 \%\end{array}$ & $\begin{array}{l}1 / 7 \\
14 \%\end{array}$ & $\begin{array}{l}1 / 7 \\
14 \%\end{array}$ & $\begin{array}{r}0 / 7 \\
0 \%\end{array}$ & $\begin{array}{r}0 / 7 \\
0 \%\end{array}$ \\
\hline
\end{tabular}

generation Sikhs and Hindus were found to be similar to that of their first generation elders.

The Muslim community was found to have very low alcohol usage, with $92 \%$ of 1 st generation Muslims and $94 \%$ of 2 nd generation Muslims claiming not to drink any alcohol. Alcohol use was also low in the Jain community, where $82 \%$ of the sample group

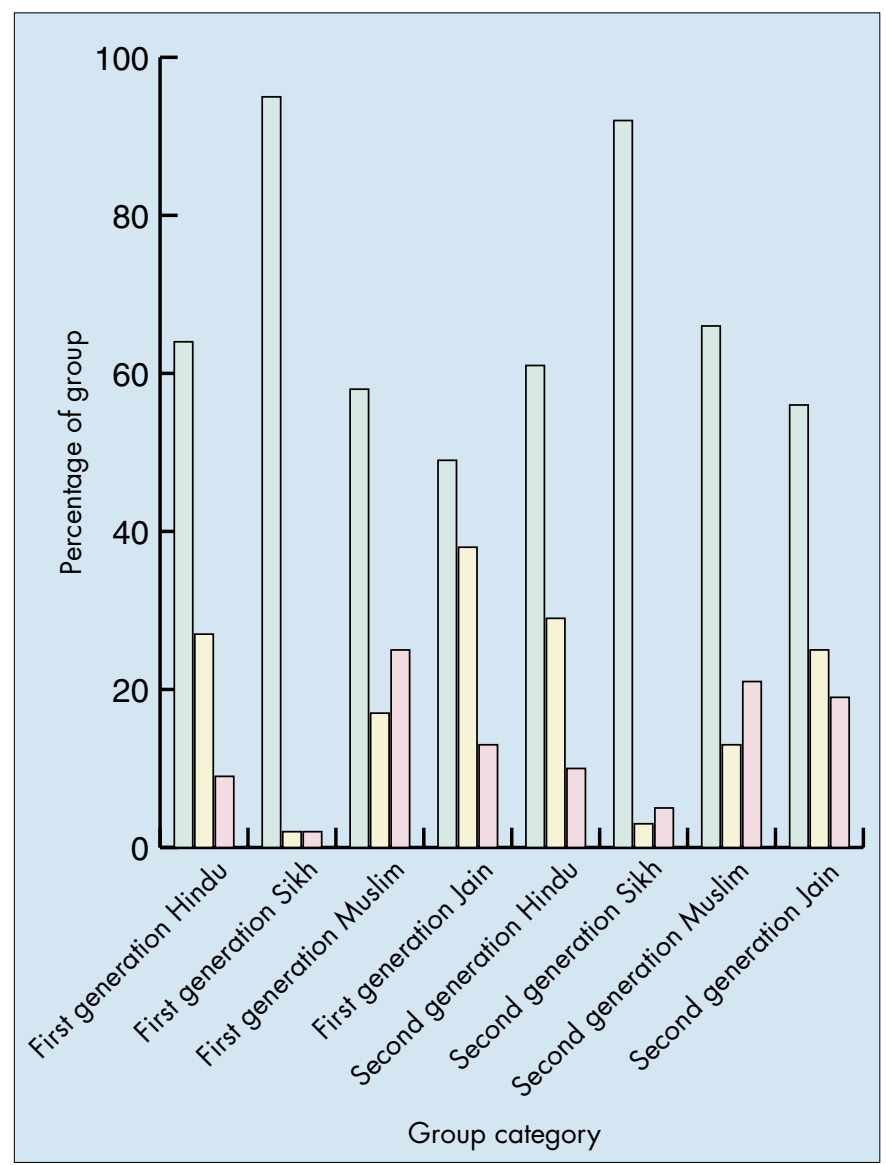

Fig. 3 Paan use among Leicester Asian males in study group. Green, non-chewers; yellow, occasionally chew; red, regularly chew. stated 'no' to drinking any alcohol. However, a statistically significant increase was observed in the number of second generation Jains (44\%) drinking alcohol regularly compared with first generation Jains $(13 \%)\left(\chi^{2}, \mathrm{p}<0.001\right)$.

Beer and lager were the main types of alcohol drunk by alcohol users in all communities, and in both 1st and 2nd generation groups, being drunk by $43 \%$ of 1 st generation Jain drinkers to $88 \%$ of Sikh drinkers. Fifty two per cent of 1st generation Sikh drinkers also drank spirits.

A higher proportion of 2nd generation Hindu and Sikh males were drinking spirits compared with their 1st generation counterparts (Table 2).

The drinking of 'alcopops' was only found among the 2nd generation groups.

Tobacco Use

Among 1st generation males, the high use of tobacco observed among Muslim males compared with the other groups was significant $\left(\chi^{2}, p<0.001\right)$ (Fig. 2, Table 3). Forty four percent of Muslim males used tobacco regularly, mainly in the form of cigarettes, in contrast to the low tobacco usage by 1 st generation Sikhs. Ninety five percent of the Sikh group stated that they did not use tobacco.

Slight differences among the 2nd generation sample group were not statistically significant.

The small increase in the number of 2 nd generation Hindu and Sikh males smoking tobacco compared with their first generation counterparts was not statistically significant (Table 3 ). However, the decrease in the number of 2 nd generation Muslim males smoking tobacco compared with their elders was significant $\left(\chi^{2}, \mathrm{p}<0.05\right)$.

\section{Paan Use}

Considerable differences exist in both 1st and 2nd generation Asian populations regarding the chewing of paan $\left(\chi^{2}, \mathrm{p}<0.001\right)$ (Fig. 3$)$.

Highest numbers of regular paan users were found in the 1st generation Muslim community, with $25 \%$ of the sample group claiming to chew paan regularly and a further $17 \%$ on an occasional basis (Table 4).

This is in contrast to the low usage of paan among 1st generation Sikhs, where only $2.5 \%$ were regular paan chewers. 


\section{oral cancer}

Table 5 Sopari (areca nut) and paan masala use among Leicester Asian males in the study group

\begin{tabular}{|c|c|c|c|c|c|c|}
\hline Sample Group & $\begin{array}{l}\text { Sopari use } \\
\text { No }\end{array}$ & Occasional & Yes & $\begin{array}{l}\text { Paan masala use } \\
\text { No }\end{array}$ & Occasional & Yes \\
\hline \multicolumn{7}{|c|}{ First generation } \\
\hline Hindu $(n=105)$ & $\begin{array}{r}74 \\
71 \%\end{array}$ & $\begin{array}{r}12 \\
11 \%\end{array}$ & $\begin{array}{r}19 \\
18 \%\end{array}$ & $\begin{array}{r}95 \\
90 \%\end{array}$ & $\begin{array}{r}6 \\
6 \%\end{array}$ & $\begin{array}{r}4 \\
4 \%\end{array}$ \\
\hline Sikh $(n=40)$ & $\begin{array}{r}40 \\
100 \%\end{array}$ & $\begin{array}{r}0 \\
0 \%\end{array}$ & $\begin{array}{r}0 \\
0 \%\end{array}$ & $\begin{array}{r}39 \\
97.5 \%\end{array}$ & $\begin{array}{r}1 \\
2.5 \%\end{array}$ & $\begin{array}{r}0 \\
0 \%\end{array}$ \\
\hline Jain $(n=39)$ & $\begin{array}{r}21 \\
54 \%\end{array}$ & $\begin{array}{r}7 \\
18 \%\end{array}$ & $\begin{array}{r}11 \\
28 \%\end{array}$ & $\begin{array}{r}36 \\
92 \%\end{array}$ & $\begin{array}{r}0 \\
0 \%\end{array}$ & $\begin{array}{r}3 \\
8 \%\end{array}$ \\
\hline \multicolumn{7}{|c|}{ Second generation } \\
\hline Hindu $(n=157)$ & $\begin{array}{l}114 \\
73 \%\end{array}$ & $\begin{array}{r}23 \\
14 \%\end{array}$ & $\begin{array}{r}20 \\
13 \%\end{array}$ & $\begin{array}{l}145 \\
92 \%\end{array}$ & $\begin{array}{r}5 \\
3 \%\end{array}$ & $\begin{array}{r}7 \\
5 \%\end{array}$ \\
\hline Sikh $(n=61)$ & $\begin{array}{r}59 \\
97 \%\end{array}$ & $0 \%$ & $\begin{array}{r}2 \\
3 \%\end{array}$ & $\begin{array}{r}59 \\
97 \%\end{array}$ & $0 \%$ & $\begin{array}{r}2 \\
3 \%\end{array}$ \\
\hline Muslim (n=53) & $\begin{array}{r}34 \\
64 \%\end{array}$ & $\begin{array}{r}10 \\
19 \%\end{array}$ & $\begin{array}{r}9 \\
17 \%\end{array}$ & $\begin{array}{r}49 \\
92 \%\end{array}$ & $0 \%$ & $\begin{array}{r}4 \\
8 \%\end{array}$ \\
\hline Jain $(n=16)$ & $\begin{array}{r}12 \\
75 \%\end{array}$ & $\begin{array}{r}2 \\
12.5 \%\end{array}$ & $\begin{array}{r}2 \\
12.5 \%\end{array}$ & $\begin{array}{r}14 \\
88 \%\end{array}$ & $\begin{array}{c}1 \\
6 \%\end{array}$ & $\begin{array}{r}1 \\
6 \%\end{array}$ \\
\hline
\end{tabular}

The chewing of paan is also prevalent among 2 nd generation Hindus, Muslims and Jains, with high numbers of regular users among Muslim and Jain males. Again, low usage was observed among Sikhs.

The most common types of paan chewed by 1 st generation males were sweet paan and khari paan (containing lime) (Table 4). Twenty one per cent of Hindu paan users chewed paan containing tobacco.

However, among 2nd generation males, the majority of paans chewed were of the sweet variety. This change as to the type of paan chewed between 1st and 2 nd generation Hindu males was found to be statistically significant $\left(\chi^{2}, \mathrm{p}<0.001\right)$.

The chewing of paans containing tobacco and/or lime, though, is still practised by a few 2 nd generation males.

\section{Areca Nut Use}

Significant differences regarding the chewing of areca nut alone was noted among the 1 st generation $\left(\chi^{2}, \mathrm{p}<0.001\right)$ and 2 nd generation groups $\left(\chi^{2}, \mathrm{p}<0.01\right)$ (Table 5$)$.

Among 1st generation males, highest numbers of areca nut chewers were found among the Jain community, where $28 \%$ chewed areca nut regularly with a further $18 \%$ on an occasional basis. Twenty three percent of Muslim and 18\% of Hindu males were regular areca nut chewers. Similar to the results of paan chewing, use of areca nut by Sikh males was absent.

Among the 2nd generation sample, highest use was by Muslim males where $17 \%$ regularly chewed areca nut; compared with $13 \%$ of Hindu and $12.5 \%$ of Jain males. Use of areca nut by Sikhs was low,

Table 6 Combined habits among Leicester Asian males in the study group

(Chewing habit includes paan, and/or areca nut, and/or paan masala chewing)

\begin{tabular}{|c|c|c|c|c|c|c|c|c|}
\hline \multirow[t]{2}{*}{ Sample Group } & \multicolumn{2}{|c|}{$\begin{array}{l}\text { No. of People } \\
\text { with no habit }\end{array}$} & \multicolumn{3}{|c|}{ Single Habit } & \multicolumn{2}{|l|}{ Two Habits } & Three Habits \\
\hline & & $\begin{array}{l}\text { Alcohol } \\
\text { only }\end{array}$ & $\begin{array}{l}\text { Tobacco } \\
\text { only }\end{array}$ & $\begin{array}{l}\text { Chewing } \\
\text { only }\end{array}$ & $\begin{array}{r}\text { Tobacco } \\
+ \text { alcohol }\end{array}$ & $\begin{aligned} & \text { Alcohol } \\
+ & \text { chewing }\end{aligned}$ & $\begin{array}{r}\text { Tobacco } \\
+ \text { chewing }\end{array}$ & $\begin{array}{l}\text { Tobacco + alcohol } \\
\quad+\text { chewing }\end{array}$ \\
\hline \multicolumn{9}{|c|}{ First Generation } \\
\hline Hindu $(n=105)$ & $\begin{array}{r}29 \\
27.6 \%\end{array}$ & $\begin{array}{r}21 \\
20 \%\end{array}$ & $\begin{array}{r}0 \\
0 \%\end{array}$ & $\begin{array}{r}14 \\
13.3 \%\end{array}$ & $\begin{array}{r}10 \\
9.5 \%\end{array}$ & $\begin{array}{r}21 \\
20 \%\end{array}$ & $\begin{array}{r}0 \\
0 \%\end{array}$ & $\begin{array}{r}10 \\
9.5 \%\end{array}$ \\
\hline Sikh $(n=40)$ & $\begin{array}{r}14 \\
35 \%\end{array}$ & $\begin{array}{r}22 \\
55 \%\end{array}$ & $\begin{array}{r}0 \\
0 \%\end{array}$ & $\begin{array}{r}1 \\
2.5 \%\end{array}$ & $\begin{array}{r}2 \\
5 \%\end{array}$ & $\begin{array}{r}1 \\
2.5 \%\end{array}$ & $\begin{array}{r}0 \\
0 \%\end{array}$ & $\begin{array}{r}0 \\
0 \%\end{array}$ \\
\hline Muslim(n=48) & $\begin{array}{r}12 \\
25 \%\end{array}$ & $\begin{array}{r}2 \\
4.2 \%\end{array}$ & $\begin{array}{r}11 \\
22.9 \%\end{array}$ & $\begin{array}{r}8 \\
16.7 \%\end{array}$ & $\begin{array}{r}0 \\
0 \%\end{array}$ & $\begin{array}{r}0 \\
0 \%\end{array}$ & $\begin{array}{r}13 \\
27.1 \%\end{array}$ & $\begin{array}{r}2 \\
4.2 \%\end{array}$ \\
\hline Jain $(n=39)$ & $\begin{array}{r}11 \\
28.2 \%\end{array}$ & $\begin{array}{r}2 \\
5.1 \%\end{array}$ & $2.6 \%$ & $\begin{array}{r}17 \\
43.6 \%\end{array}$ & $2.6 \%$ & $\begin{array}{r}4 \\
10.3 \%\end{array}$ & $\begin{array}{r}3 \\
7.7 \%\end{array}$ & $\begin{array}{r}0 \\
0 \%\end{array}$ \\
\hline \multicolumn{9}{|c|}{ Second Generation } \\
\hline Hindu $(n=157)$ & $\begin{array}{r}30 \\
19.1 \%\end{array}$ & $\begin{array}{r}35 \\
22.3 \%\end{array}$ & $\begin{array}{r}1 \\
0.6 \%\end{array}$ & $\begin{array}{r}16 \\
10.2 \%\end{array}$ & $\begin{array}{r}21 \\
13.4 \%\end{array}$ & $\begin{array}{r}34 \\
21.7 \%\end{array}$ & $\begin{array}{r}0 \\
0 \%\end{array}$ & $\begin{array}{r}20 \\
12.7 \%\end{array}$ \\
\hline Sikh $(n=61)$ & $\begin{array}{r}24 \\
39.3 \%\end{array}$ & $\begin{array}{r}23 \\
37.7 \%\end{array}$ & $\begin{array}{r}0 \\
0 \%\end{array}$ & $\begin{array}{r}4 \\
6.6 \%\end{array}$ & $\begin{array}{r}9 \\
14.8 \%\end{array}$ & $\begin{array}{r}1 \\
1.6 \%\end{array}$ & $\begin{array}{r}0 \\
0 \%\end{array}$ & $\begin{array}{r}0 \\
0 \%\end{array}$ \\
\hline Muslim (n=53) & $\begin{array}{r}18 \\
33.9 \%\end{array}$ & $\begin{array}{r}2 \\
3.8 \%\end{array}$ & $\begin{array}{r}8 \\
15.1 \%\end{array}$ & $\begin{array}{r}17 \\
32.1 \%\end{array}$ & $\begin{array}{r}0 \\
0 \%\end{array}$ & $\begin{array}{r}0 \\
0 \%\end{array}$ & $\begin{array}{r}7 \\
13.2 \%\end{array}$ & $\begin{array}{r}1 \\
1.9 \%\end{array}$ \\
\hline Jain $(n=16)$ & $\begin{array}{r}3 \\
18.8 \%\end{array}$ & $\begin{array}{r}5 \\
31.3 \%\end{array}$ & $\begin{array}{r}0 \\
0 \%\end{array}$ & $\begin{array}{r}3 \\
18.8 \%\end{array}$ & $\begin{array}{r}1 \\
6.3 \%\end{array}$ & $\begin{array}{r}3 \\
18.8 \%\end{array}$ & $\begin{array}{r}0 \\
0 \%\end{array}$ & $\begin{array}{r}1 \\
6.3 \%\end{array}$ \\
\hline
\end{tabular}


Table 7 Oral cancer awareness among Leicester Asian males in study group

\begin{tabular}{|c|c|c|c|c|c|c|c|c|}
\hline \multirow[t]{2}{*}{ Sample group } & \multicolumn{2}{|c|}{ Heard of oral cancer? } & \multicolumn{6}{|c|}{ Source of knowledge of oral cancer } \\
\hline & Yes & No & $\begin{array}{l}\text { School/ } \\
\text { College }\end{array}$ & $\begin{array}{c}\text { Press/TV/ } \\
\text { Media }\end{array}$ & $\begin{array}{c}\text { Doctor/ } \\
\text { Dentist advice }\end{array}$ & $\begin{array}{c}\text { Health education } \\
\text { leaflets }\end{array}$ & $\begin{array}{l}\text { Know someone } \\
\text { with oral cancer }\end{array}$ & Other \\
\hline \multicolumn{9}{|c|}{ First generation } \\
\hline Hindu $(n=105)$ & $\begin{array}{r}57 \\
54 \%\end{array}$ & $\begin{array}{r}48 \\
46 \%\end{array}$ & $\begin{array}{r}5 / 57 \\
9 \%\end{array}$ & $\begin{array}{r}42 / 57 \\
74 \%\end{array}$ & $\begin{array}{r}6 / 57 \\
11 \%\end{array}$ & $\begin{array}{r}9 / 57 \\
16 \%\end{array}$ & $\begin{array}{r}6 / 57 \\
11 \%\end{array}$ & $\begin{array}{r}0 / 57 \\
0 \%\end{array}$ \\
\hline Muslim (n=48) & $\begin{array}{r}22 \\
46 \%\end{array}$ & $\begin{array}{r}26 \\
54 \%\end{array}$ & $\begin{array}{r}4 / 22 \\
18 \%\end{array}$ & $\begin{array}{r}11 / 22 \\
50 \%\end{array}$ & $\begin{array}{r}6 / 22 \\
27 \%\end{array}$ & $\begin{array}{r}3 / 22 \\
14 \%\end{array}$ & $\begin{array}{r}2 / 22 \\
9 \%\end{array}$ & $\begin{array}{r}0 / 22 \\
0 \%\end{array}$ \\
\hline $\begin{array}{l}\text { Jain }(n=39) \\
\text { Second gener }\end{array}$ & $\begin{array}{r}20 \\
51 \% \\
\end{array}$ & $\begin{array}{r}19 \\
49 \%\end{array}$ & $\begin{array}{r}1 / 20 \\
5 \%\end{array}$ & $\begin{array}{r}10 / 20 \\
50 \%\end{array}$ & $\begin{array}{r}7 / 20 \\
35 \%\end{array}$ & $\begin{array}{r}3 / 20 \\
15 \%\end{array}$ & $\begin{array}{r}2 / 20 \\
10 \%\end{array}$ & $\begin{array}{r}0 / 20 \\
0 \%\end{array}$ \\
\hline Hindu $(n=157)$ & $\begin{array}{r}67 \\
43 \%\end{array}$ & $\begin{array}{r}90 \\
57 \%\end{array}$ & $\begin{array}{r}25 / 67 \\
37 \%\end{array}$ & $\begin{array}{r}26 / 67 \\
39 \%\end{array}$ & $\begin{array}{r}7 / 67 \\
10 \%\end{array}$ & $\begin{array}{r}15 / 67 \\
22 \%\end{array}$ & $\begin{array}{r}4 / 67 \\
6 \%\end{array}$ & $\begin{array}{r}2 / 67 \\
3 \%\end{array}$ \\
\hline Sikh $(n=61)$ & $\begin{array}{r}29 \\
48 \%\end{array}$ & $\begin{array}{r}32 \\
52 \%\end{array}$ & $\begin{array}{r}17 / 29 \\
59 \%\end{array}$ & $\begin{array}{r}13 / 29 \\
45 \%\end{array}$ & $\begin{array}{r}2 / 29 \\
7 \%\end{array}$ & $\begin{array}{r}7 / 29 \\
24 \%\end{array}$ & $\begin{array}{r}1 / 29 \\
3 \%\end{array}$ & $\begin{array}{r}0 / 29 \\
0 \%\end{array}$ \\
\hline Muslim (n=53) & $\begin{array}{r}23 \\
43 \%\end{array}$ & $\begin{array}{r}30 \\
57 \%\end{array}$ & $\begin{array}{r}13 / 23 \\
57 \%\end{array}$ & $\begin{array}{r}7 / 23 \\
30 \%\end{array}$ & $\begin{array}{r}5 / 23 \\
22 \%\end{array}$ & $\begin{array}{r}7 / 23 \\
30 \%\end{array}$ & $\begin{array}{r}1 / 23 \\
4 \%\end{array}$ & $\begin{array}{r}0 / 23 \\
0 \%\end{array}$ \\
\hline Jain $(n=16)$ & $\begin{array}{r}12 \\
75 \%\end{array}$ & $\begin{array}{r}4 \\
25 \%\end{array}$ & $\begin{array}{r}6 / 12 \\
50 \%\end{array}$ & $\begin{array}{r}5 / 12 \\
42 \%\end{array}$ & $\begin{array}{r}4 / 12 \\
33 \%\end{array}$ & $\begin{array}{r}4 / 12 \\
33 \%\end{array}$ & $\begin{array}{r}1 / 12 \\
8 \%\end{array}$ & $\begin{array}{r}0 / 12 \\
0 \%\end{array}$ \\
\hline
\end{tabular}

with only $3 \%$ of the group being regular chewers.

\section{Paan Masala Use}

The use of paan masala among 1st generation males, either on a regular or occasional basis, was noted in $15 \%$ of Muslim, $10 \%$ of Hindu and $8 \%$ of Jain males (Table 5). Chewing of paan masala was lowest in the Sikh community, with $97.5 \%$ of the sample group stating that they did not use paan masala. This again reflects the finding of a low prevalence of a chewing habit among Sikh males.

Among 2nd generation males, a similar picture of use was observed with $12 \%$ of Jains, $8 \%$ of Hindus and $8 \%$ of Muslims chewing paan masala. Again, use among the Sikh group was low, with $97 \%$ not partaking in the habit.

\section{Combined Habits}

Table 6 summarises all the habits of the study group.

Among 1st generation males, 9.5\% of Hindu males combined all 3 habits of tobacco, alcohol and chewing paan or areca nut. A further $20 \%$ combined a chewing habit with alcohol use.

Twenty seven percent of Muslim males combined their chewing habit with tobacco smoking.

A high proportion of Sikhs (55\%) drank alcohol as their only habit. Forty three point six per cent of Jains undertook a solely chewing habit.

Approximately $30 \%$ of 1 st generation males did not partake in any habit.

Among the 2nd generation group, a similar picture of combining habits was noticed in Hindu males, where $12.7 \%$ of the sample group combined all 3 habits. Twenty two percent of the group combined both alcohol drinking and a chewing habit.

Thirty two percent of Muslim males had a chewing habit alone, and $37.7 \%$ and $31.3 \%$ of Sikh and Jain males respectively drank alcohol as their only habit.

In contrast to approximately $40 \%$ of 2 nd generation Sikh males, under $20 \%$ of Hindu and Jain males had no habit.

\section{Oral Cancer Awareness among Asian Males in Leicester}

Among 1st generation Asian males, approximately 50\% had heard of oral cancer, except for the Sikh group where only $17.5 \%$ of the sample group had heard of the condition (Table 7). This difference was highly significant $\left(\chi^{2}, \mathrm{p}<0.001\right)$.
Regarding the source of their knowledge, over $50 \%$ of Hindu, Jain and Muslim males stated that they had heard of oral cancer from the press and media. Approximately 10\% of those who had heard of oral cancer knew of someone with the condition.

Seventy five percent of 2 nd generation Jains had heard of oral cancer, compared with $43-48 \%$ in the other 2 nd generation groups $\left(\chi^{2}, \mathrm{p}<0.1\right)$. A greater proportion of 2 nd generation Sikhs $(48 \%)$ had heard of oral cancer compared with their first generation elders $(17.5 \%)\left(\chi^{2}, \mathrm{p}<0.01\right)$.

Major sources of knowledge for 2 nd generation males included school/college education, the press and media, and health education leaflets.

When 1st generation males were asked about risk factors implicated in the aetiology of oral cancer, significant differences were observed. Seventy eight percent of Sikh males did not know or gave no response, compared with around $50 \%$ for the other community groups $\left(\chi^{2}, \mathrm{p}<0.01\right)$.

Paan and areca nut chewing, and tobacco smoking were frequently given as causative risk factors for oral cancer by around $30 \%$ of Hindu, Muslim and Jain males; but fewer (3-8\%) stated that alcohol was a risk factor.

A lower proportion of 2nd generation Sikhs (54\%) gave no response for oral cancer risk factors compared with their elders (77.5\% no response) $\left(\chi^{2}, p<0.05\right)$. Approximately one third of the Hindu, Sikh and Muslim; and 50\% of Jain males stated that smoking was a risk factor, followed by paan chewing. Again, only around $10 \%$ stated that alcohol was a potential risk factor.

With regards to the prevention of oral cancer development, a large proportion of 1st generation Sikhs (67.5\%) gave no response/did not know, which was higher than the other community groups $(43-54 \%)\left(\chi^{2}, \mathrm{p}<0.1\right)$. Second generation Sikhs gave a better response rate compared with their elders (60\% and 32.5\% respectively giving a response) $\left(\chi^{2}, \mathrm{p}<0.01\right)$.

Approximately $30 \%$ of 1 st and 2 nd generation males stated stopping tobacco smoking, stopping paan/areca nut chewing and keeping the mouth clean could help prevent oral cancer developing. Only around $10 \%$ stated that reducing alcohol consumption would help.

\section{Discussion}

Our study confirms that the U.K. 'Asian' population is not homogeneous, but is composed of distinct community groups such as 
Hindu, Sikh, Muslim and Jain. In addition, significant differences in habits and oral cancer awareness exist between these groups.

Alcohol use was found to be highest among both 1st and 2nd generation Sikh males. Although mean units of alcohol drank per week may be lower than that for Caucasians, some Hindu and Sikh males did have a weekly alcohol consumption considered to be high (over 21 units/week). ${ }^{12}$ The prohibition of alcohol use in the Islamic and Jain faiths is demonstrated by the very low use of alcohol in the 1st generation groups studied. However, despite low alcohol consumption being continued among 2nd generation Muslims, a significant increase in alcohol drinkers was observed among 2nd generation Jains.

Beer and lager were the main types of alcohol drunk by both 1st and 2 nd generation males. However, it was observed that a greater proportion of 2 nd generation male drinkers were drinking spirits and 'alcopops' compared with their 1st generation elders. The frequent drinking of spirits among the young Hindu and Sikh males may be a cause for concern for future levels of drinking as these men become older.

In contrast to their low alcohol use, Muslim groups had the highest usage of tobacco, mainly in the form of cigarettes, compared with the other Hindu, Sikh and Jain groups. Tobacco smoking was found to be relatively infrequent among Sikhs. Similar findings have been reported in the Health Education Authority's Health and Lifestyle Survey of Black and Minority Ethnic Groups. ${ }^{11}$

The chewing of paan was found to be highest among the 1st generation Muslim group, and was also prevalent at a level of 30-40\% among 1st and 2nd generation Hindu and Jain males. Very low usage of paan among Sikh males was noticed.

Although the levels of paan chewing in this study are not as high as the $90 \%$ and over prevalence rates among the Bangladeshi communities in Yorkshire ${ }^{3}$ and Birmingham; ${ }^{15}$ the moderate levels of chewing found in the Leicester population probably reflects the levels of chewing practised in the Indian states of Gujarat, Punjab and Rajasthan where rates varying from $4-30 \%$ have been reported. ${ }^{11,23}$

Although sweet paans are generally chewed by 2 nd generation males, more frequent use of khari paans, containing lime, and paans containing tobacco by 1st generation paan chewers was demonstrated. Twelve percent of 1 st generation Hindu males were found in this study to chew lime-containing paan, and 7\% chewed paan containing tobacco. Both types of paan are associated with an increased carcinogenic risk compared with plain or sweet paans containing areca nut alone. ${ }^{4,23,25}$

The risk of squamous cell carcinoma developing in the oral cavity is increased by the combining of habits of alcohol drinking, tobacco smoking and paan use..$^{25,26}$ Such combining of habits was frequently observed among 1st and 2nd generation Hindu and Muslim males. First generation Muslim males frequently combined tobacco smoking with the chewing of paan and/or areca nut.

Combination of all 3 habits was found in approximately $10 \%$ of 1 st and 2 nd generation Hindu males. This group should be considered to be at high risk for the development of oral cancer.

Levels of knowledge about oral cancer among the participants in this study were generally lower than that found in a study of 400 patients in a medical practice where $65 \%$ had heard of oral cancer. ${ }^{38}$ Few 1st generation Sikhs had heard of the condition, compared with about half of the respondants from the other groups studied.

Knowledge regarding causative and preventive factors for oral cancer was again lowest among 1st generation Sikhs. For the other groups, approximately a third knew that smoking and paan/areca nut chewing were risk factors for oral cancer development; and that stopping these habits were preventive measures. However, few respondants recognised alcohol as a risk factor and that by reducing intake could help prevent oral cancer development. Similar findings of a low awareness of the carcinogenic risk of high alcohol intake has been reported elsewhere. ${ }^{38}$ Hence, education regarding the risks of alcohol use, as well as the other risk factors, needs to be improved; especially since increasing numbers of 2 nd generation Hindus, Sikhs and Jains are drinking alcohol — spirits in particular.

\section{Conclusion}

This study has shown that the Asian population in Leicester is not a homogeneous group. Significant differences exist with regards to alcohol, tobacco and paan use among Hindus, Sikhs, Muslims and Jains; and also between 1st and 2nd generation males.

Sikh males tend to drink alcohol, whereas Muslim males use tobacco and chew paan Around 10\% of Hindu males combine all three habits, and are considered to be at high risk of oral cancer development.

Knowledge regarding oral cancer risk factors and preventive measures was found to be variable, with lowest levels among 1st generation Sikhs. Few people in all the community groups realised the risk of alcohol drinking in the aetiology of oral cancer.

Awareness of the variations in risk factor use and knowledge regarding oral cancer that exist within Leicester's Asian population, can be used to provide health education programmes appropriately targeted to the needs of the different community groups.

The authors would like to thank: all the volunteers and centres that participated in the study; Mrs Prafulla Vora and Dr Gautam Bodiwala for their assistance in the translation of the questionnaire.

1 Blot W J, McLaughlin J K, Winn D M et al. Smoking and drinking in relation to oral and pharyngeal cancer. Cancer Res 1988; 48: 3282-3287.

2 Brugere J, Guevel P, Leclerc A, Rodriguez J. Differential effects of tobacco and alcohol in cancer of the larynx, pharynx and mouth. Cancer 1986; 57: 391-395.

3 Summers R M, Williams S A, Curzon M E J. The use of tobacco and betel quid ('pan') among Bangladeshi women in West Yorkshire. Community Dent Health 1994; 11: 12-16.

4 Gupta PC, Nandakumar A. Oral cancer scene in India. Oral Dis 1999; 5: 1-

5 Vokes E E, Weichselbaum R R, Lippman S M, Hong W K. Head and Neck Cancer. New Eng J Med 1993; 328: 184-194.

6 Llewelyn J, Mitchell R. Smoking, alcohol and oral cancer in South East Scotland: a 10 year experience. Br J Oral Maxillofac Surg 1994; 32: 146152

7 EU-Working Group on Tobacco and Oral Health - Meeting Report. Oral Dis 1998; 4: 48-67.

8 Hindle I, Downer M C, Speight M. The epidemiology of oral cancer. Br J Oral Maxillofac Surg 1996; 34: 471-476.

9 Ogden G R, Wight A J. Aetiology of oral cancer. Br J Oral Maxillofac Surg 1998; 36: 247-251.

10 A J Wight, Ogden G R. Possible mechanisms by which alcohol may influence the development of oral cancer - a review. Eur J Cancer B Oral Oncol 1998; 34: 441-447.

11 Black and Minority Ethnic Groups in England - Health and Lifestyles Survey, Chapter 5: Smoking, pp 78-100, London: Health Education Authority, 1994.

12 Bennet N, Dodd T, Flatley J, Freeth S, Bolling K. Health Survey for England 1993. London, HMSO, 1993.

13 Sloan D, Goepfert H. Conventional therapy of head and neck cancer. Hematology/Oncol Clin N Am 1991; 5: 601-625

14 van der Waal I. Tobacco and oral cancer and pre-cancer. Oral Dis 1998; 4: 52-53.

15 Bedi R. Betel-quid and tobacco chewing among the United Kingdom's Bangladeshi community. Br J Cancer 1996; 74 Suppl. XXIX: S73-S77.

16 Merchant N E, Ferguson M M, Ali A, Hole D J, Gillis C R. Oral carcinoma in the Indian and Pakistani community in Scotland. J Oral Med 1986; 41: 62-65.

17 Donaldson L J, Clayton D G. Occurrence of cancer in Asians and nonAsians. J Epidemiol Community Health 1984; 38: 203-207.

18 Swerdlow A J, Marmot M G, Gruhlich A E, Head J. Cancer mortality in Indian and British ethnic immigrants from the Indian subcontinent to England and Wales. Br J Cancer 1995; 72: 1312-1319.

19 Barker R M, Baker M R. Incidence of cancer in Bradford Asians. J Epidemiol Community Health 1990; 44: 125-129.

20 Balarajan R, Bulusu L, Adelstein A M, Shukla V. Patterns of mortality 
among migrants to England and Wales from the Indian subcontinent. $\mathrm{Br}$ Med J 1984; 289: 1185-1187.

21 Warnakulasuriya K A A S, Johnson N W. Epidemiology and risk factors for oral cancer: Rising trends in Europe and possible effects of migration. Int Dent J 1996; 46(Suppl 1): 245-50.

22 Zain R B, Ikeda N, Gupta P C et al. Oral mucosal lesions associated with betel quid, areca nut and tobacco chewing habits: consensus from a workshop held in Kuala Lumpur, Malaysia, November 25-27, 1996. J Oral Pathol Med 1999; 28;1-4.

23 International Agency for Research on Cancer. IARC monographs on the evaluation of the carcinogenic risk of chemicals to humans - Vol 37: Tobacco habits other than smoking; betel-quid and areca nut chewing; and some related nitrosamines. World Health Organisation, Lyon, France, 1985.

24 Nair U J, Obe J, Friesen M, Goldberg M T, Bartsch H. The role of lime in the generation of reactive oxygen species from betel quid ingredients. Environ Health Perspect 1992; 98: 203-205.

25 Thomas S, Kearsley J. Betel quid and oral cancer: A review. Eur J Cancer B Oral Oncol 1993; 29B: 251-255.

26 Ko Y C, Huang Y L, Lee C H, Chen M J, Lin L M, Tsai C C. Betel quid chewing, cigarette smoking and alcohol consumption related to oral cancer in Taiwan. J Oral Pathol Med 1995; 24: 450-453.

27 Murti P R, Bhonsle R B, Gupta P C, Daftary D K, Pindborg J J, Mehta F S. Etiology of oral submucous fibrosis with special reference to the role of areca nut chewing. J Oral Pathol Med 1995; 24: 145-152.
28 Oral submucous fibrosis: An expert symposium. Meeting report. Oral Dis 1997; 3: 276-291.

29 Patel R K, Trivedi A H, Jaju R J, Adhvaryu S G, Balar D B. Ethanol potentiates the clastogenicity of pan masala - an in vitro experience. Carcinogenesis 1994; 15: 2017-2021.

30 Bahl V. Cancer and ethnic minorities - the Department of Health's perspective. Br J Cancer 1996; 74 Suppl. XXIX: S2-S10.

31 Bedi R, Uppal R D K. The oral health of minority ethnic groups in the United Kingdom. Br Dent J 1995; 179: 421-425.

32 Balarajan R, Soni Raleigh V. The ethnic populations of England and Wales: the 1991 Census. Health Trends 1992; 24: 113-116.

33 Policy Studies Institute. Ethnic Minorities in Britain: Diversity and Disadvantage. The Fourth National Survey of Ethnic Minorities. London: Policy Studies Institute, 1997, PSI Report 843.

34 Williams S A, Ahmed I A, Hussain P. Ethnicity, health and dental care perspectives among British Asians: 1. Dent Update 1991; 18: 154-161.

35 Bedi R. Betel-quid - worldwide use. Oral Dis 1997; 3: 284-286.

36 Feehally J, Burden A C, Mayberry J F, Probert C S J, Roshan M, Samanta A K, Woods K L. Disease variations in Asians in Leicester. Quart J Med 1993; 86: 263-269.

37 Leicester City Council/Leicestershire County Council. 1983 Survey of Leicester. Leicester: Leicestershire County Council, 1984.

38 Zakrzewska J M. Oral cancer and precancer - our responsibility. Br Dent J 1994; 176: 286-287. 